\title{
Iterative Training of Unsupervised Neural and Statistical Machine Translation Systems
}

\author{
BENJAMIN MARIE and ATSUSHI FUJITA, National Institute of Information and Communications \\ Technology, Japan
}

\begin{abstract}
Recent work achieved remarkable results in training neural machine translation (NMT) systems in a fully unsupervised way, with new and dedicated architectures that only rely on monolingual corpora. However, previous work also showed that unsupervised statistical machine translation (USMT) performs better than unsupervised NMT (UNMT), especially for distant language pairs. To take advantage of the superiority of USMT over UNMT, and considering that SMT suffers from well-known limitations overcome by NMT, we propose to define UNMT as NMT trained with the supervision of synthetic parallel data generated by USMT. This way we can exploit USMT up to its limits while ultimately relying on full-fledged NMT models to generate translations. We show significant improvements in translation quality over previous work and also that further improvements can be obtained by alternatively and iteratively training USMT and UNMT. Without the need of a dedicated architecture for UNMT, our simple approach can straightforwardly benefit from any recent and future advances in supervised NMT. Our systems achieve a new state-of-the-art for unsupervised machine translation in all of our six translation tasks for five diverse language pairs, surpassing even supervised SMT or NMT in some tasks. Furthermore, our analysis shows how crucial the comparability between the monolingual corpora used for unsupervised training is in improving translation quality.
\end{abstract}

\section{CCS Concepts: • Computing methodologies $\rightarrow$ Machine translation;}

Additional Key Words and Phrases: Machine translation, phrase table induction, low-resource language pair, semantic similarity, knowledge acquisition

\section{ACM Reference format:}

Benjamin Marie and Atsushi Fujita. 2020. Iterative Training of Unsupervised Neural and Statistical Machine Translation Systems. ACM Trans. Asian Low-Resour. Lang. Inf. Process. 19, 5, Article 68 (May 2020), 21 pages. https://doi.org/10.1145/3389790

\section{INTRODUCTION}

Machine translation (MT) systems usually require a large amount of bilingual parallel data, produced by humans, as supervision for training. However, finding such data remains challenging for most language pairs, as it may not exist or may be too costly to manually produce.

In contrast, a large amount of monolingual data can be easily collected for many languages. Previous work proposed many ways for taking advantage of monolingual data in order to improve

Authors' address: B. Marie and A. Fujita, National Institute of Information and Communications Technology, 3-5 Hikaridai, Seika-cho, Soraku-gun, Kyoto 619-0289, Japan; emails: \{bmarie, atsushi.fujita\}@nict.go.jp.

Permission to make digital or hard copies of all or part of this work for personal or classroom use is granted without fee provided that copies are not made or distributed for profit or commercial advantage and that copies bear this notice and the full citation on the first page. Copyrights for components of this work owned by others than the author(s) must be honored. Abstracting with credit is permitted. To copy otherwise, or republish, to post on servers or to redistribute to lists, requires prior specific permission and/or a fee. Request permissions from permissions@acm.org.

(C) 2020 Copyright held by the owner/author(s). Publication rights licensed to ACM.

2375-4699/2020/05-ART68 \$15.00

https://doi.org/10.1145/3389790 
translation models trained on parallel data $[12,34]$. These methods usually exploit existing accurate translation models to generate synthetic parallel data that can be used to augment the training data of the NMT systems. Recent work has also shown remarkable results in training MT systems using only monolingual data in the source and target languages in so-called unsupervised statistical (USMT) and neural (UNMT) machine translation [2, 5, 24]. However, existing approaches for UNMT rely on dedicated architectures significantly different from the well-established architectures designed for supervised NMT. Existing UNMT architectures relatively remain unoptimized and under-explored with many specific hyper-parameters difficult to set. On the other hand, running existing USMT only requires slight modifications of well-known SMT frameworks and consistently outperforms UNMT in translation quality as demonstrated by Artetxe et al. [2]. Nonetheless, SMT architecture has well-known limits that are overcome by NMT. Exploiting an NMT architecture for unsupervised MT seems intuitive to perform as close as possible to supervised MT.

Following our promising preliminary experiments presented in [26], we propose, in order to exploit USMT up to its limits while ultimately relying on full-fledged NMT models, a new approach for unsupervised MT in which USMT and UNMT are assisting each other through an iterative training, only using synthetic parallel data for supervision. We answer the following research questions:

- Can synthetic bilingual data generated by a given unsupervised MT system be used to train from scratch an MT system better than the given unsupervised MT system?

- How do the language pairs and the comparability between the monolingual corpora impact on the translation quality in unsupervised MT?

In our way toward answering these questions, we report on the following outcomes:

- We propose a simplified USMT framework, which is easier to set up and train than the existing frameworks.

- We propose to cast UNMT with a standard NMT framework by simply replacing manually produced parallel data with synthetic parallel data generated by USMT. This pseudosupervision strategy enables us to use well-established and well-optimized NMT architectures with all their features, without assuming any relatedness between targeted languages in contrast to previous work.

- We propose a new unsupervised iterative training approach in which USMT and UNMT assist each other for training.

- We demonstrate that the feasibility of unsupervised MT depends largely on the language pair and the comparability of the monolingual corpora used.

The remainder of this article is organized as follows. We first discuss in Section 2 how we define "unsupervised." In Section 3, we introduce our architecture for USMT and then in Section 4 we present our approach for an UNMT trained on synthetic parallel data. In Section 5, we describe our approach to combine the advantages of USMT and UNMT using an iterative training framework. In Section 6, we evaluate our USMT, UNMT, and their iterative training on six translation tasks and present an analysis of the results. We conduct further experiments and analyses in Section 7 , in settings where we have more comparable monolingual corpora to train our systems. Section 8 exposes the concurrent work and highlights strengths and weaknesses of other approaches for unsupervised MT. Finally, Section 9 concludes this article.

\section{WHAT IS UNSUPERVISED IN THIS ARTICLE?}

Since the term "unsupervised" may be ambiguous, we present in this section what aspects of this work are assumed to be unsupervised. 


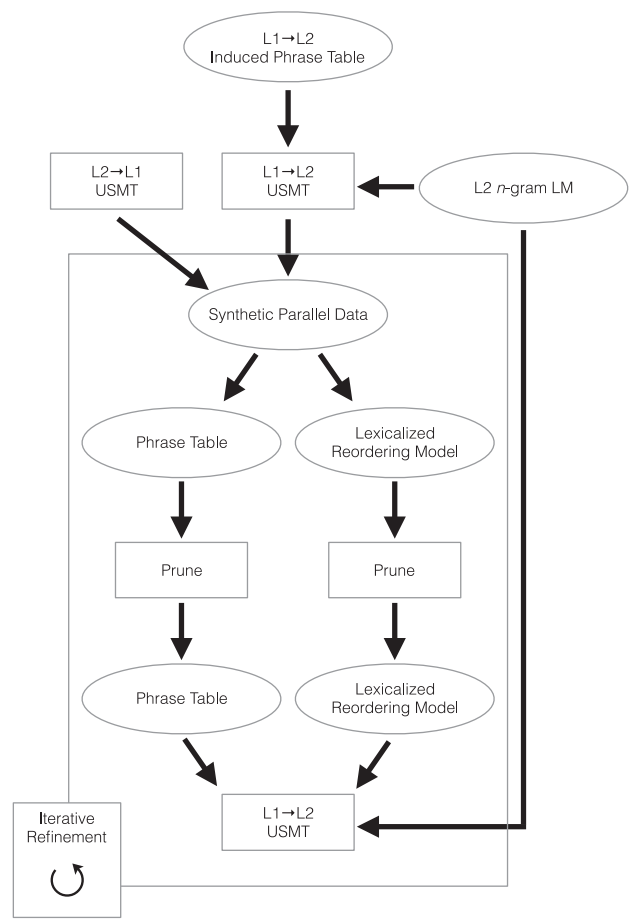

Fig. 1. Our USMT framework for the translation direction $\mathrm{L} 1 \rightarrow \mathrm{L} 2$ of a given language pair $\mathrm{L} 1-\mathrm{L} 2$. It is analogous for $\mathrm{L} 2 \rightarrow \mathrm{L} 1$.

As previous work, we define "unsupervised MT" as MT that does not use manually produced translation pairs as parallel data for training. Nonetheless, MT still needs some supervision for training and we use synthetic parallel data generated from monolingual data for this purpose.

In this article, "unsupervised" only qualifies the training of MT systems on parallel data of which at least one side is synthetic. For tuning, i.e., finding the best model weights for USMT or selecting the best model for UNMT, it is supervised in all our experiments using a small development set of manually produced sentence pairs. We assume that this development set can be inexpensive to create manually or automatically. Nonetheless, we discuss "unsupervised tuning" in Section 3.2 and report on experimental results in Section 6.5. For evaluation, it must be fully supervised since we use a manually produced test set to evaluate the translation quality.

Even if our systems are trained without manually produced parallel data, we can still argue that the monolingual corpora used to generate synthetic parallel data have been produced by humans. Monolingual corpora in our experiments (see Section 6.1) could include comparable parts that could have a significant impact on the translation quality. Moreover, we cannot ensure that they do not contain any human-made translations from which our systems can take advantage during training. Finally, we use SMT and NMT architectures with their hyper-parameters (for instance, the default parameters of the Transformer model [39]) that have already shown to give good results in MT.

\section{USMT}

Our USMT framework, as illustrated in Figure 1, is based on the same architecture proposed by previous work $[2,24]$ : a phrase table is induced from monolingual data and used to compose the 
initial USMT system that is then refined iteratively using synthetic parallel data. We propose various modifications in this section to better ground each step required to set up an USMT system. An ablation study is reported in Section 6.4 to evaluate the impact of our modifications.

\subsection{Phrase Table Induction}

In USMT, phrase table induction is regarded as the process of creating a phrase table using only monolingual resources. It takes as inputs a set of phrases in the source language, another set of phrases in the target language, and their respective embeddings.

3.1.1 Previous Work. Artetxe et al. [2] regarded the most frequent unigrams, bigrams, and trigrams in the monolingual data as phrases. The embedding of each $n$-gram is computed with a generalization of the skipgram algorithm [27]. Then, source and target $n$-gram embedding spaces are aligned in the same bilingual embedding space without supervision [1]. Lample et al. [24]'s method also works at $n$-gram level using the method by Mikolov et al. [27 $]^{1}$ that was also used by Marie and Fujita [25] in the context of phrase table induction.

3.1.2 Phrase Collection. We induce our phrase tables with phrases collected as in Marie and Fujita [25]. This method can collect more meaningful phrases than only the most frequent $n$ grams [2]. Whereas Mikolov et al. [27]'s phrase collection method was originally proposed to identify non-compositional phrases, we rather choose to enforce the collection of more compositional phrases, ${ }^{2}$ for which embeddings are easier to estimate, because of the following reasons:

- Very few phrases are non-compositional in standard SMT systems [41].

- Most of them are not very frequent.

-Useful representation of compositional phrases can easily be obtained compositionally [43].

Consequently, we obtain a set of phrases mainly made of compositional phrases for which phrase embeddings can be compositionally computed [24, 43].

3.1.3 Phrase Pair Scoring. To obtain the pairs of source and target phrases that populate the induced phrase table, we use the equation proposed by Lample et al. [24]: ${ }^{3}$

$$
p\left(t_{j} \mid s_{i}\right)=\frac{\exp \left(\beta \cos \left(e m b\left(t_{j}\right), e m b\left(s_{i}\right)\right)\right)}{\sum_{k} \exp \left(\beta \cos \left(e m b\left(t_{k}\right), e m b\left(s_{i}\right)\right)\right)},
$$

where $t_{j}$ is the $j$-th phrase in the target phrase list, $s_{i}$ the $i$-th phrase in the source phrase list, $\beta$ a parameter to tune the peakiness of the distribution [35], ${ }^{4}$ and $e m b(\cdot)$ a function returning the bilingual embedding of a given phrase. $p\left(s_{i} \mid t_{j}\right)$ is computed analogously for the other translation direction.

We also compute two lexical translation probabilities for each phrase pair, using the equation proposed by Song and Roth [37] that computes a similarity score between two phrases, i.e.,

\footnotetext{
${ }^{1}$ The exact method used is not mentioned in the article and is not in the publicly released implementation. We found this information at https:/github.com/facebookresearch/UnsupervisedMT/issues/64\#issuecomment-471700200. However, note that we could not confirm whether it was used for the experiments of the paper [24].

${ }^{2}$ This is done by using a low value for the parameter $\delta$, the discount coefficient in Equation (6) in Mikolov et al. [27], which prevents us from collecting infrequent phrases. We set $\delta=10$ in all our experiments.

${ }^{3}$ We could not obtain results similar to the results reported in Lample et al. [24] by using their Equation (3) with $\beta=30$ as they proposed. We have confirmed through personal communications with the authors that Equation (1), as we wrote, with $\beta=30$, generates the expected results. We do not use the equation computing $\phi$ in Artetxe et al. [2], since it produces a negative value as a probability when cosine similarity is negative.

${ }^{4} \mathrm{We}$ set $\beta=30$ since it is the default value proposed in the code released by Smith et al. [35]: https://github.com/ Babylonpartners/fastText_multilingual.
} 
sequences of words, given word embeddings:

$$
\operatorname{lex}\left(t_{j} \mid s_{i}\right)=\frac{1}{L} \prod_{l=1}^{L} \max _{k=1}^{K} p\left(t_{j}^{k} \mid s_{i}^{l}\right),
$$

where $K$ and $L$ are the number of words in $t_{j}$ and $s_{i}$, respectively, and $p\left(t_{j}^{k} \mid s_{i}^{l}\right)$ the translation probability of the $k$-th target word $t_{j}^{k}$ of $t_{j}$ given the $l$-th source word $s_{i}^{l}$ of $s_{i}$ given by Equation (1). $\operatorname{lex}\left(s_{i} \mid t_{j}\right)$ is computed analogously for the other translation direction. Note that, unlike Song and Roth [37] and Kajiwara and Komachi [18], we do not use a threshold value under which $p\left(t_{j}^{k} \mid s_{i}^{l}\right)$ is ignored, since it would be costly to tune for each translation task.

After the computation of the above four scores for each phrase pair in the induced phrase table, the phrase table is plugged in an SMT system to perform what we denote in the remainder of this article as step 0 of USMT. ${ }^{5}$

\subsection{Discussion about Unsupervised Tuning}

State-of-the-art supervised SMT performs the weighted log-linear combination of different models [28] for decoding. The model weights are tuned given a small development set of sentence pairs. For fully unsupervised SMT, we cannot assume the availability of this development set. Model weights must be tuned without the supervision of manually produced parallel data.

Lample et al. [24] used some pre-existing default weights that work reasonably well. On the other hand, Artetxe et al. [2] used 10,000 monolingual sentences paired with their back-translations as a development set. Nonetheless, to create this development set, they also relied on the same preexisting default weights used by Lample et al. [24]: the default weights of the Moses framework [20].

However, regarding the use of default weights as "unsupervised tuning" is arguable, since these default weights have been manually determined for European languages. For translation between very distant languages, these default weights would likely result in a very poor translation quality. We argue that unsupervised tuning remains one of the main issues in current approaches for USMT.

While manually creating large training parallel data for a particular language pair is very costly, which is one of the fundamental motivations of unsupervised MT, we can assume that a small set of sentence pairs ${ }^{6}$ required for tuning is already available ${ }^{7}$ or can be created at a reasonable cost. In this work, we present results with supervised tuning only and leave for future work the study of unsupervised methods for generating development data. For a comparison, we present results of USMT and our whole framework without using any development set in Section 6.5.

\subsection{Refinement}

3.3.1 Previous Work. Artetxe et al. [2] and Lample et al. [24] proposed so-called refinement steps. Those steps use USMT to generate synthetic parallel data to train a new phrase table, with refined translation probabilities. This can be repeated for several iterations to improve USMT. The initial system at step 0 uses the induced phrase table (see Section 3.1), while the following steps

\footnotetext{
${ }^{5}$ Klementiev et al. [19] showed that lexicalized reordering models for the phrase pairs in the induced phrase table can be computed from monolingual data, and they are potentially helpful. However, for the sake of simplicity, we do not compute these lexical reordering models for step 0 .

${ }^{6}$ For instance, the WikiMatrix project (https://github.com/facebookresearch/LASER/tree/master/tasks/WikiMatrix) proposes a small set of sentence pairs for 1,620 language pairs extracted from Wikipedia that could be used for tuning purposes. ${ }^{7}$ As in the unsupervised MT shared task of WMT19: http://www.statmt.org/wmt19/.
} 
use only a phrase table and a reordering model trained on the synthetic parallel data generated by USMT.

In previous work, for a given language pair L1-L2, the synthetic parallel data were generated through back-translation: the L2 $\rightarrow$ L1 USMT system was used to back-translate L2 sentences. Then, L2 sentences paired with their translations in L1 were used as synthetic parallel data to train a new $\mathrm{L} 1 \rightarrow \mathrm{L} 2 \mathrm{USMT}$ system. Using back-translation in SMT has been proven useful by previous work [21]. It has also been proposed to improve NMT systems [34] with a specific motivation to enhance the decoder by exploiting fluent sentences in L2. However, using only back-translation for training USMT lacks motivation. Since the source side of the synthetic parallel data, i.e., decoded results of USMT, tends to not be fluent, USMT will learn a phrase table with many ungrammatical L1 phrases or untranslated words in L2 that will never be seen in L1, meaning that many phrase pairs in the phrase table will never be used while skewing the translation probability distribution. Moreover, possible and frequent source phrases, or even source words, may not be generated via back-translation and will be consequently absent from the trained phrase table.

3.3.2 Refinement with Forward Translations. To ensure that the phrase tables will contain fluent target phrases, we keep using back-translations for the refinement steps. Nevertheless, to compensate for the shortcomings of exclusive use of back-translations, we also use synthetic parallel data made of L1 sentences translated into L2 by the L1 $\rightarrow$ L2 USMT system, i.e., "forward translation." Actually, the idea of retraining an SMT system on synthetic parallel data generated by an L1 $\rightarrow$ L2 system has already been proven beneficial [38].

At each iteration, we randomly sample $Q \mathrm{~L} 1$ and $Q \mathrm{~L} 2$ sentences from their respective monolingual corpus and translate them with the latest $\mathrm{L} 1 \rightarrow \mathrm{L} 2$ and $\mathrm{L} 2 \rightarrow \mathrm{L} 1$ USMT systems, respectively, to generate a total of $2 Q$ synthetic sentence pairs that can be used to train both $\mathrm{L} 1 \rightarrow \mathrm{L} 2$ and $\mathrm{L} 2 \rightarrow \mathrm{L} 1$ USMT systems. In contrast, using only either back-translation or forward translation requires twice the computational cost for generating both of them independently with $\mathrm{L} 2 \rightarrow \mathrm{L} 1$ and $\mathrm{L} 1 \rightarrow \mathrm{L} 2$ systems, in order to obtain two different sets of $2 Q$ synthetic sentence pairs for both translation directions.

3.3.3 Phrase Table Pruning. Since we train phrase tables on noisy synthetic parallel data, we prune the phrase tables as proposed by Johnson et al. [16] to remove less reliable phrase pairs without any significant drop of the translation quality. This pruning also speeds up decoding for generating synthetic parallel data for the next step since numerous translation options are removed in advance.

\section{UNMT AS NMT TRAINED EXCLUSIVELY ON SYNTHETIC PARALLEL DATA}

\subsection{Previous Work}

Many methods $[4,23,24,40]$ proposed dedicated architectures for UNMT, such as denoising autoencoders, shared latent representations, weight sharing, pre-trained embeddings, and adversarial training. However, as the following and concurrent studies [2, 24] have demonstrated, existing UNMT frameworks hardly realize systems significantly better than USMT systems, especially in translation tasks for which SMT performs well.

\subsection{Training UNMT on Synthetic Parallel Data}

We propose to train UNMT systems exclusively on synthetic parallel data, simply using existing frameworks for supervised NMT without any modification. Our approach is substantially different from the "PBSMT+NMT" configuration in Lample et al. [24] in the following three aspects. 


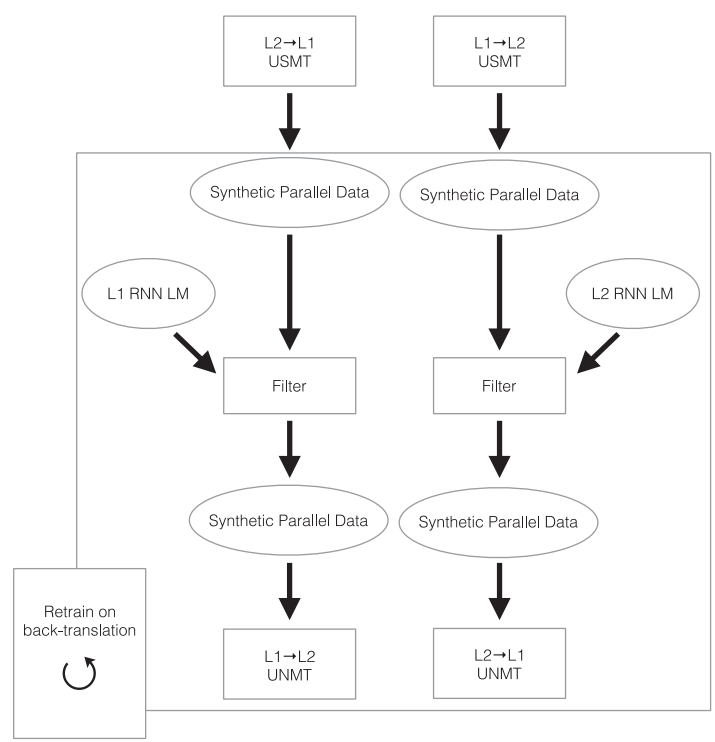

Fig. 2. Our UNMT framework for a given language pair L1-L2.

(1) While their method uses synthetic parallel data generated by USMT only to further tune their dedicated UNMT architecture, ours uses it as ordinary parallel data for training NMT.

(2) They assumed a certain level of relatedness between languages, which is a prerequisite to jointly pre-train bilingual sub-word embeddings. Our approach does not make this assumption.

(3) We do not rely on a particular architecture and therefore are free to use any available NMT frameworks, maximally benefiting from their up-to-date features and efficiency.

\subsection{Back-Translation and Data Filtering}

To obtain better UNMT systems, we propose the following procedure (see also Figure 2).

Assuming that USMT produces translations of a reasonable quality, we first train $\mathrm{L} 1 \rightarrow \mathrm{L} 2$ and L2 $\rightarrow$ L1 UNMT systems on $Q$ sentence pairs respectively generated by back-translating monolingual data with $\mathrm{L} 2 \rightarrow \mathrm{L} 1$ and $\mathrm{L} 1 \rightarrow \mathrm{L} 2 \mathrm{USMT}$ systems. Since USMT may generate very ungrammatical translations, we only keep for training the $\alpha \cdot Q(0<\alpha \leq 1)^{8}$ sentence pairs with the most fluent translations generated by USMT according to a normalized language model score:

$$
\operatorname{ppl}(S)=\frac{\operatorname{lm}(S)}{\operatorname{len}(S)+1},
$$

where $S$ is an output of USMT, i.e., synthetic source sentence, $\operatorname{lm}(\cdot)$ the language model score, and len $(\cdot)$ a function returning the number of tokens in the sentence. We add 1 to the denominator to account for the special token used by NMT that marks the end of a sentence.

There are many ways to make use of the above score during NMT training. For instance, weighting the sentence pairs with this score during training is a possible alternative, and this idea is close to the joint training framework for NMT proposed by Cheng et al. [8]. However, given that many of the synthetic sentence pairs would be noisy, we rather choose to exclude potentially noisy pairs for training. We also expect this filtering to significantly speed up convergence during training since it uses smaller training data.

\footnotetext{
${ }^{8}$ This ratio was arbitrarily set to 0.5 in all our experiments.
} 


\subsection{Refinement}

To fully exploit the potential of NMT in generating more fluent sentences than SMT, especially for morphologically rich languages and for language pairs requiring long-distance reordering, we retrain from scratch the $\mathrm{L} 1 \rightarrow \mathrm{L} 2$ UNMT system on $P$ synthetic parallel sentences generated by back-translating L1 sentences using the L2 $\rightarrow$ L1 UNMT system, mixed with those generated by the L2 $\rightarrow$ L1 USMT system. The entire set of synthetic parallel sentences is also filtered in the same manner as in Section 4.3 to keep the best $\alpha \cdot(P+Q)$ sentence pairs for training.

\section{ITERATIVE TRAINING OF USMT AND UNMT}

In contrast to supervised NMT where synthetic parallel data are added to manually produced parallel data, we can presumably use as much synthetic parallel data as possible, since seeing more fluent target sentences will help training a better UNMT decoder while we can assume that the quality of the synthetic source side remains constant. In practice, since the decoder focuses on the head of the model distribution, it leads to back-translations likely to be very regular by favoring the same translations in case of ambiguity and with a poor coverage of the true data distribution [12]. Generating a larger quantity of synthetic parallel data is also costly and provides only limited improvements, if any, as also confirmed by previous work on USMT by Lample et al. [24, Figure 3].

We propose an alternative framework to improve our USMT and UNMT systems iteratively. Assuming that our approach for UNMT can generate translations of a better quality than the translations generated by USMT, we train a new USMT system on synthetic parallel data generated by UNMT and expect to obtain an USMT system better than the previous USMT system. This procedure can be repeated for several iterations to improve USMT and UNMT. Within each iteration, USMT and UNMT are trained from scratch to make sure that they are not biased by the synthetic data generated earlier, already used for previous training, and presumably of a lower quality. The training of UNMT is always initialized by synthetic parallel data generated by the last USMT system, while the training of USMT is initialized by synthetic parallel data generated by the UNMT system of the previous iteration, except for the first iteration for which USMT is initialized with an induced phrase table (Section 3.1).

The number of iterations to perform is pre-set and the best MT systems, among all the systems trained, can be selected using a development set.

\section{EXPERIMENTS}

In order to evaluate the feasibility and the performance of our USMT, UNMT, and of their iterative training, we conducted experiments in various settings, including high-resource and low-resource language pairs.

\subsection{Experimental Settings}

We experimented with five language pairs: English-German (en-de), English-French (en-fr), English-Japanese (en-ja), English-Indonesian (en-id), and Japanese-Indonesian (ja-id). We performed six translation tasks only using monolingual corpora for training, development data for tuning USMT and for model selection of UNMT, and test data for evaluation, presented in Table 1.

For en-de and en-fr, we used the same data used in previous work [24] to conduct comparable experiments. Since the monolingual corpora are clean and comparable to some degree, we can expect a reasonable translation quality. We can have the same expectation for en-ja-NTCIR; even though this task involves much more distant languages, the difficulty of training unsupervised bilingual word embeddings is alleviated by the comparability of the source and target monolingual corpora [36]. Moreover, a good coverage of the dev/test sets by the induced phrase tables is ensured, 
Table 1. Data Used in Our Experiments for Each Translation Task

\begin{tabular}{|c|c|c|c|}
\hline \multirow{2}{*}{$\begin{array}{l}\text { Language pair } \\
\text { (L1-L2-task) }\end{array}$} & \multicolumn{2}{|c|}{ Monolingual corpora (domain) } & dev/test \\
\hline & (\#tokens in L1) & (\#tokens in L2) & (domain) \\
\hline \multirow{2}{*}{ en-de-news } & \multicolumn{2}{|c|}{ News Crawl (newswire) } & Newstest2015/Newstest2016 \\
\hline & $(2007-2017,5.3 \mathrm{~B})$ & $(2007-2017,4.3 \mathrm{~B})$ & (newswire) \\
\hline \multirow{2}{*}{ en-fr-news } & \multicolumn{2}{|c|}{ News Crawl (newswire) } & Newstest2013/Newstest2014 \\
\hline & $(2007-2017,5.3 \mathrm{~B})$ & $(2007-2014,0.9 \mathrm{~B})$ & (newswire) \\
\hline \multirow[t]{2}{*}{ en-ja-NTCIR } & \multicolumn{2}{|c|}{ NTCIR (patent) } & NTCIR dev/test-10 \\
\hline & $(3.0 \mathrm{~B})$ & (3.9 B) & (patent) \\
\hline \multirow[t]{2}{*}{ en-id-ALT } & \multicolumn{2}{|c|}{ Common Crawl (any) } & ALT dev/test \\
\hline & $(1.8 \mathrm{~B})$ & $(2.2 \mathrm{~B})$ & (newswire) \\
\hline \multirow[t]{2}{*}{ en-ja-ALT } & \multicolumn{2}{|c|}{ Common Crawl (any) } & ALT dev/test \\
\hline & $(1.8 \mathrm{~B})$ & $(1.9 \mathrm{~B})$ & (newswire) \\
\hline \multirow[t]{2}{*}{ ja-id-ALT } & \multicolumn{2}{|c|}{ Common Crawl (any) } & ALT dev/test \\
\hline & $(1.9 \mathrm{~B})$ & $(2.2 \mathrm{~B})$ & (newswire) \\
\hline
\end{tabular}

News Crawl corpora, English Common Crawl, and Newstest data are provided by the Conference on Machine Translation [7]. NTCIR data are provided by the NTCIR patent translation task [13]. We obtained the Common Crawl corpora for Japanese and Indonesian from the official Web site. ALT dev/test are provided by the Asian Language Treebank project [32].

since the monolingual corpora and the dev/test sets belong to the same domain. In contrast, the three ALT tasks are expected to be much more difficult, since the involved monolingual data, i.e., Common Crawl corpora, ${ }^{9}$ are essentially crawled Web pages from any domain and thus potentially extremely noisy. ja-id and en-id can be considered as extremely low-resource language pairs for which unsupervised MT may have applications.

All our English, German, French, and Indonesian data were pre-processed with the tokenizer and truecaser of Moses. ${ }^{10}$ The statistics for truecasing were learned from $10 \mathrm{M}$ sentences randomly sampled from the monolingual data. We tokenized Japanese data with MeCab. ${ }^{11}$

For the phrase table induction, the source and target word embeddings were learned from the entire monolingual data with the default parameters of fastText [6], ${ }^{12}$ except that we set the number of dimensions to 512. We retained only the embeddings for the 300,000 most frequent words, following previous work, in order to limit computational cost. Word embeddings for each pair of languages were then aligned in the same space using the --unsupervised option of vecmap. ${ }^{13}$ From the entire monolingual data, we collected phrases of up to six tokens in each language using word2phrase, ${ }^{14}$ but retained only the 300,000 most frequent phrases made of words among the 300,000 most frequent words in the corresponding monolingual data for a reasonably fast computation. We also conserved only the 300-best target phrases for each source phrase according to Equation (1), resulting in an induced phrase table for USMT containing $90 \mathrm{M}(300,000 \times 300)$ phrase pairs. ${ }^{15}$

\footnotetext{
$\overline{{ }^{9} \text { http://commoncrawl.org/. }}$

${ }^{10}$ https://github.com/moses-smt/mosesdecoder.

${ }^{11}$ https://taku910.github.io/mecab/.

${ }^{12}$ https://fasttext.cc/.

${ }^{13}$ https://github.com/artetxem/vecmap.

${ }^{14}$ https://code.google.com/archive/p/word2vec/.

${ }^{15}$ We intended to reduce the significant computational cost for computing $p\left(s_{i} \mid t_{j}\right)$, $\operatorname{lex}\left(t_{j} \mid s_{i}\right)$, and $\operatorname{lex}\left(s_{i} \mid t_{j}\right)$. Using many more phrases, as in Artetxe et al. [2], can significantly improve coverage and potentially translation quality.
} 
We used Moses and its default parameters to conduct experiments for USMT with four refinement steps. At each step, we sampled 1.5 M monolingual sentences from the source and target monolingual corpora, i.e., $Q=1.5 \times 10^{6}$, and translate them, using cube-pruning for fast decoding [15], to generate synthetic parallel data. We then trained a phrase table and a lexicalized reordering model on the synthetic parallel data using fast_align [11]. ${ }^{16}$ We used 4-gram language models trained with LMPLZ [14] on the entire monolingual data. For tuning, we used kb-mira [9].

For UNMT, we used the Transformer [39] model implemented in Marian [17] ${ }^{17}$ with the hyperparameters proposed by Vaswani et al. [39] but using only four layers to speed up training and decoding. We reduced the vocabulary size by using byte pair encoding (BPE) with 8,000 sub-words independently learned on $10 \mathrm{M}$ sentences randomly sampled from each set of monolingual data. $\mathrm{BPE}$ was then applied to the entire source and target monolingual data. ${ }^{18}$ For training, $3 Q$ sentences were sampled from monolingual data $(P=3 Q)$, back-translated, and then the resulting synthetic parallel data were mixed with the $Q$ sentence pairs generated by the last iteration of USMT. The data were filtered according to an RNN language mode ${ }^{19}$ to keep only $2 Q(=\alpha \cdot(P+Q)$, with $\alpha=$ 0.5 and $P=3 Q$ ) sentence pairs for training. During training, our models were validated according to their perplexity computed on the development set.

We report on results for two iterations of iterative training for USMT and UNMT. We performed only two refinement steps for training USMT at iteration 2 since we did not observe any noticeable improvements on the development data when performing more steps. Note also that each iteration of UNMT used the same size of synthetic parallel data for training, i.e., $Q$ and $P$ remained constant.

Our baseline USMT and (Transformer-based) UNMT systems were respectively trained on 3 $\mathrm{M}$ back-translated data, generated from sentences randomly sampled, at each refinement step, and on $10 \mathrm{M}$ sentences sampled from source and target monolingual corpora, with the original implementation of Lample et al. [24]. ${ }^{20}$ For these systems, we also exploited the development data for supervised tuning and validation. However, despite our best efforts, we could not reproduce the results of their "PBSMT+NMT" configuration. ${ }^{21}$

For reference, we also trained supervised SMT and NMT, with Moses and Marian, respectively, on 5.6 M, 26.7 M, and 3.1 M sentence pairs, ${ }^{22}$ respectively, for the en-de, en-fr, and en-ja-NTCIR tasks. For the ALT tasks, we used the $18 \mathrm{k}$ parallel sentences provided for training.

We evaluated our systems with detokenized and detruecased BLEU-cased [29] for all languages except for Japanese. Note that our results should not be directly compared with the tokenized BLEU scores reported in previous work, as discussed in Post [30]. ${ }^{23}$ For Japanese, we report on tokenized BLEU-cased.

\subsection{Results}

6.2.1 Translation Quality. Our results are presented in Table 2 for en-de, en-fr, and en-ja-NTCIR tasks, and in Table 3 for ALT tasks. We exclude en-ja-ALT and ja-id tasks from this part of our analysis, and will discuss them in Section 6.2.2.

\footnotetext{
${ }^{16} \mathrm{mg}$ iza may provide better results, especially for difficult language pairs [10], but for a much greater computational cost.

${ }^{17}$ https://marian-nmt.github.io/, version 1.6.

${ }^{18}$ We did not use BPE for USMT.

${ }^{19}$ Trained with Marian on the entire monolingual data, without BPE, with a vocabulary size of 100,000.

${ }^{20}$ We used the default parameters assuming that they are the parameters used for the experiments reported in their paper. We could reproduce their results for en-fr and de-en pairs with these parameters.

${ }^{21}$ Their implementation for this configuration has not been released to the best of our knowledge while the details in the paper are too scarce to re-implement it by ourselves.

${ }^{22}$ All the en-de and en-fr parallel data provided by WMT, except the ParaCrawl corpus, and all the en-ja parallel data provided by NTCIR.

${ }^{23}$ For instance for French, tokenized BLEU computes scores are several BLEU points higher than detokenized BLEU.
} 
Table 2. Results for the en-de-news, en-fr-news, and en-ja-NTCIR Tasks

\begin{tabular}{|c|c|c|c|c|c|c|c|}
\hline \multirow[t]{2}{*}{ System } & \multicolumn{4}{|c|}{ Newstest } & \multicolumn{2}{|c|}{ NTCIR } & \\
\hline & $\mathrm{de} \rightarrow \mathrm{en}$ & en $\rightarrow$ de & $\mathrm{fr} \rightarrow \mathrm{en}$ & $\mathrm{en} \rightarrow \mathrm{fr}$ & $\mathrm{ja} \rightarrow \mathrm{en}$ & $\mathrm{en} \rightarrow \mathrm{ja}$ & \# \\
\hline Lample et al. [24]'s USMT & 22.1 & 17.5 & 26.2 & 23.9 & 20.5 & 21.6 & 1 \\
\hline Lample et al. [24]'s UNMT & 20.3 & 17.0 & 23.6 & 22.9 & 15.8 & 17.2 & 2 \\
\hline USMT-1 & 23.4 & 18.8 & 26.7 & 25.3 & 21.3 & 22.0 & 3 \\
\hline$\hookrightarrow$ UNMT-1 & 29.4 & 22.8 & 28.8 & 28.1 & 25.3 & 27.8 & 4 \\
\hline$\hookrightarrow$ USMT-2 & 26.6 & 21.4 & 28.0 & 27.3 & 21.6 & 25.0 & 5 \\
\hline$\hookrightarrow$ UNMT-2 & 30.4 & 24.3 & 29.2 & 29.0 & 25.9 & 29.2 & 6 \\
\hline UNMT-1 $\left(P=8.5 \times 10^{6}\right)$ & 29.8 & 22.8 & 28.9 & 28.4 & 26.0 & 28.0 & 7 \\
\hline Supervised SMT & 30.4 & 26.4 & 35.3 & 32.7 & 27.6 & 31.3 & 8 \\
\hline Supervised NMT & 35.8 & 32.9 & 35.9 & 37.2 & 43.5 & 48.7 & 9 \\
\hline
\end{tabular}

\#1-2 are baseline systems built using the framework by Lample et al. [24]. \#3-6 are our USMT and UNMT systems after one (\#3-4) and two iterations (\#5-6). \#7 is our UNMT systems at iteration 1 with $P=8.5 \times 10^{6}$ instead of $P=4.5 \times 10^{6}$ for \#2. \#8 and \#9 are respectively SMT and NMT systems trained on manually produced parallel data.

Table 3. Results for the ALT Tasks

\begin{tabular}{|c|c|c|c|c|c|c|c|}
\hline \multirow[t]{2}{*}{ System } & \multicolumn{6}{|c|}{ ALT } & \\
\hline & $\mathrm{id} \rightarrow \mathrm{en}$ & en $\rightarrow$ id & $\mathrm{ja} \rightarrow \mathrm{en}$ & $\mathrm{en} \rightarrow \mathrm{ja}$ & $\mathrm{id} \rightarrow \mathrm{ja}$ & $\mathrm{ja} \rightarrow \mathrm{id}$ & \# \\
\hline Lample et al. [24]'s USMT & 27.3 & 22.9 & 5.0 & 5.5 & 6.0 & 4.4 & 1 \\
\hline Lample et al. [24]'s UNMT & 20.5 & 18.7 & 1.9 & 2.2 & 0.8 & 0.5 & 2 \\
\hline USMT-1 & 29.1 & 24.5 & 6.0 & 6.4 & 6.6 & 4.4 & 3 \\
\hline$\hookrightarrow$ UNMT-1 & 32.3 & 28.4 & 5.7 & 6.5 & 6.6 & 4.4 & 4 \\
\hline$\hookrightarrow$ USMT-2 & 29.6 & 28.7 & 5.2 & 6.6 & 6.2 & 4.4 & 5 \\
\hline$\hookrightarrow$ UNMT-2 & 33.0 & 29.3 & 5.4 & 6.9 & 6.9 & 4.8 & 6 \\
\hline UNMT-1 $\left(P=8.5 \times 10^{6}\right)$ & 32.0 & 28.7 & 6.0 & 6.7 & 7.0 & 5.0 & 7 \\
\hline Supervised SMT & 35.5 & 35.3 & 10.9 & 15.0 & 13.8 & 8.9 & 8 \\
\hline Supervised NMT & 21.6 & 23.4 & 7.1 & 12.1 & 9.8 & 5.0 & 9 \\
\hline
\end{tabular}

Definition of each system (\#ID) follows those in Table 2.

We confirmed that USMT (\#1, \#3) outperforms Lample et al. [24]'s UNMT (\#2) for all tasks. We also observed that our USMT (\#3) outperforms Lample et al. [24]'s USMT (\#1), mainly thanks to better refinement steps (see Section 6.3).

Our approach for UNMT (\#4) is significantly better than USMT (\#1, \#3) and \#2, demonstrating that it can successfully exploit the synthetic parallel data generated by USMT. Improvements over \#2 range from $5.2(\mathrm{fr} \rightarrow \mathrm{en}$ ) to $11.8(\mathrm{id} \rightarrow \mathrm{en})$ BLEU points, bringing unsupervised MT to a dramatically better level of translation quality than previous work. The difference between \#4 and \#2 is especially large for distant languages, such as en-ja-NTCIR and en-id, presumably due to the fact that \#2 relies on shared sub-word units that may be too rare in these language pairs. Moreover, sub-word units are not semantic units, i.e., a particular shared sub-word may have a very different meaning depending on the language, and consequently should not share the same embedding across distant languages. Lample et al. [24] observed slight drops of translation quality with their unsupervised "PBSMT+NMT" configuration, including for their en-fr task for which we used the same data. In contrast, we observed consistent and significant improvements with UNMT-1 over USMT-1.

USMT-2 (\#5) outperformed USMT-1 (\#3) for all tasks but underperformed UNMT-1 (\#4). Finally, our best results were achieved by UNMT-2 (\#6) with consistent improvements over UNMT-1 (\#4), 
ranging from $0.4(\mathrm{fr} \rightarrow \mathrm{en}$ ) to 1.5 (en $\rightarrow \mathrm{de}$ ) BLEU points. Iterative training of USMT and UNMT also brought larger improvements than using more back-translated data for training UNMT-1 (\#7).

For some of our translation tasks, our systems reached the performance of supervised SMT systems trained on millions of parallel sentences, such as for de $\rightarrow$ en. It also outperformed supervised NMT trained on much less data for the en-id pair.

6.2.2 en-ja-ALT and ja-id-ALT. We obtained only a poor translation quality for these two tasks with unsupervised MT. This is the consequence of the poor quality of the synthetic parallel data generated by USMT that are not useful for initializing UNMT. USMT remains difficult to train presumably due to the difficulty of the language pair, for which unsupervised bilingual word embeddings are difficult to train and also due to the low comparability between the monolingual corpora used for training [36] (see Section 6.6).

6.2.3 Computational Cost. We used 20 CPUs to train USMT-1 (\#3), during approximately 4 days for each task to complete all the steps of iteration 1. Training of Lample et al. [24]'s USMT (\#1) was slower due to the need of generating twice more back-translated data: it consumed around 6 days for training.

Training time of Lample et al. [24]'s UNMT (\#2) was fairly unstable, varying from less than 2 days (for all ALT tasks) to 12 days (for en-fr). On the other hand, one iteration of our approach of UNMT required between 4 and 6 days of computation for each task. One advantage of our approach is that we can use an existing well-optimized NMT framework, such as Marian that is known to be very fast thanks to its native implementation in $\mathrm{C}++$ and the possibility to use multi-GPU training and decoding. For instance, we used four GPUs for our UNMT, while the original implementation of \#2 that we used exploited only one GPU. Note also that we could further reduce the training time of our approach using other features implemented in Marian, such as decoding with many CPUs or with averaged attention [42].

\subsection{Learning Curves}

Our USMT and UNMT (\#3-6) include internal refinement steps, as described in Sections 3.3 and 4.4, respectively. Figure 3 presents the improvement of BLEU scores according to this refinement, i.e., the learning curves, for all our translation tasks. The curves for en-de, en-fr, en-ja-NTCIR, and en-id are similar. There was a remarkable improvement in translation quality between step 0 and the first refinement step of USMT. The following refinement steps improved translation quality to a lesser extent, but we observed that they were more effective in our USMT framework than the refinement steps of Lample et al. [24]'s USMT (\#1). For our UNMT systems, the first system trained on back-translations generated by UNMT brought the largest improvements in most of our tasks, while the first step of UNMT led to a slight drop of translation quality, especially when translating into English.

The first refinement step of USMT-2 resulted in a significant drop of translation quality compared to UNMT-1. The second refinement step of USMT-2 did not bring improvements, or further worsened the translation quality. However, it surpassed USMT-1 in most tasks. By successfully exploiting the new outputs of USMT, subsequently, UNMT-2 recovered from the drop of translation quality.

\subsection{Ablation Study for USMT}

In this section, we present an ablation study to highlight the impact of our choices in our USMT pipeline that differentiate our systems from those in previous work. We evaluated the impact of the following. 


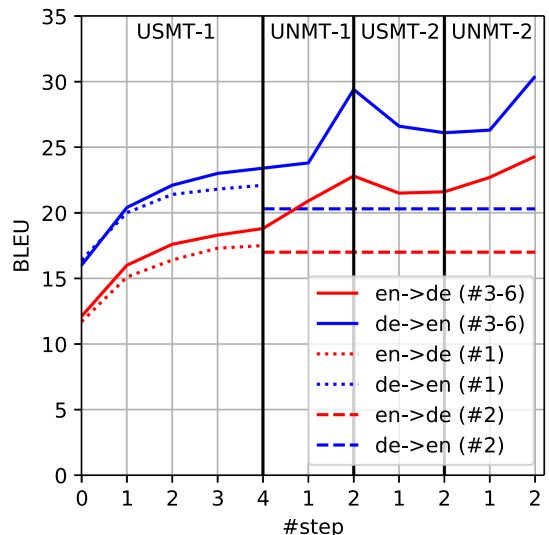

(a) en-de-news

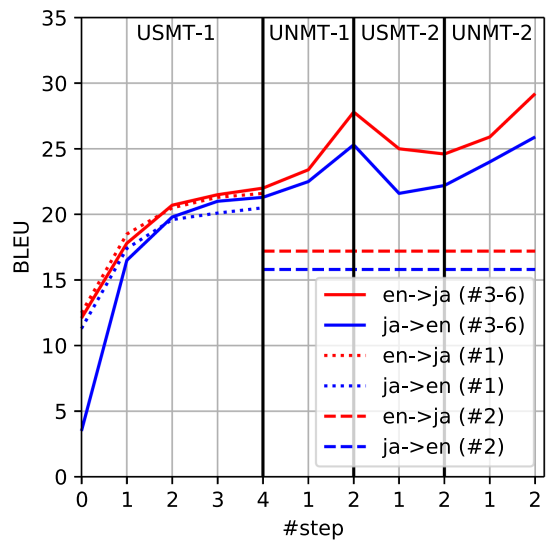

(c) en-ja-NTCIR

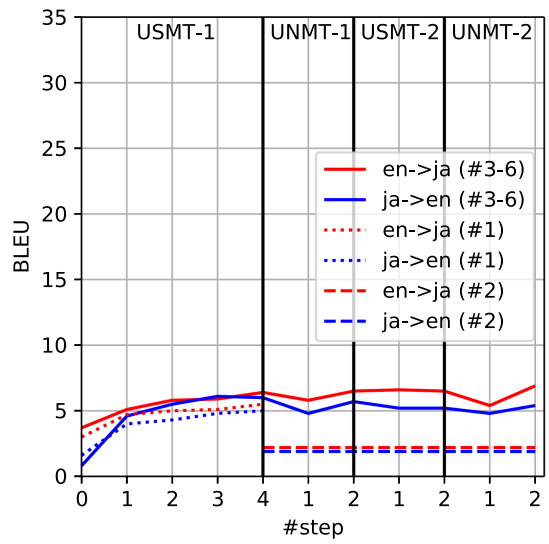

(e) en-ja-ALT

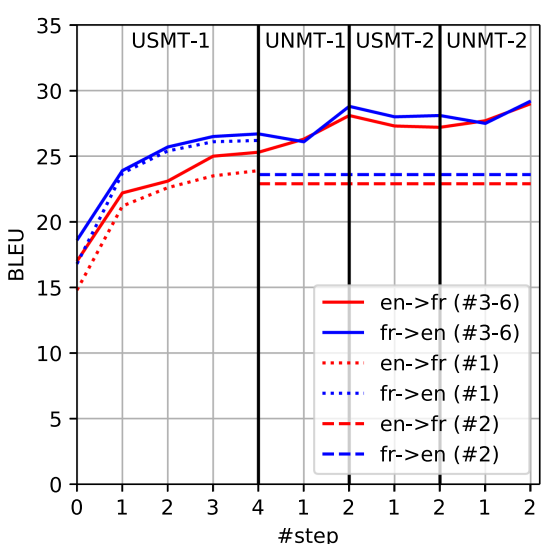

(b) en-fr-news

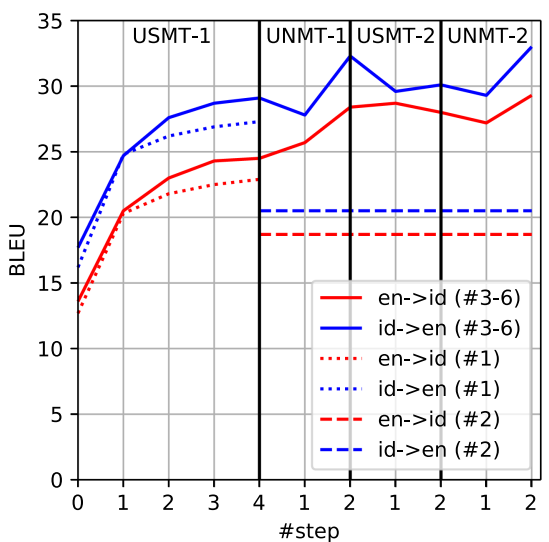

(d) en-id-ALT

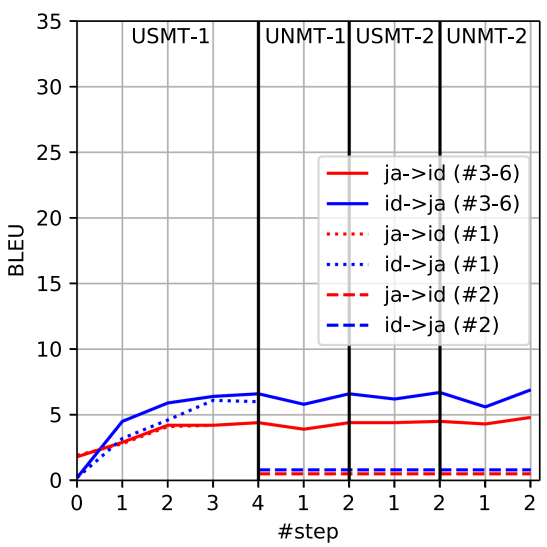

(f) ja-id-ALT

Fig. 3. Learning curves for all our tasks. \#ID refers to the systems presented in Tables 2 and 3. 
Table 4. BLEU Scores of Our Ablation Study for USMT

\begin{tabular}{|c|c|c|c|c|c|c|c|c|}
\hline \multirow{2}{*}{\multicolumn{2}{|c|}{ Configuration }} & \multirow[t]{2}{*}{ System } & \multicolumn{2}{|c|}{ en-de } & \multicolumn{2}{|c|}{ en-fr } & \multicolumn{2}{|c|}{ en-ja-NTCIR } \\
\hline & & & $\mathrm{de} \rightarrow \mathrm{en}$ & en $\rightarrow$ de & $\mathrm{fr} \rightarrow \mathrm{en}$ & $\mathrm{en} \rightarrow \mathrm{fr}$ & $\mathrm{ja} \rightarrow \mathrm{en}$ & $\mathrm{en} \rightarrow \mathrm{ja}$ \\
\hline \multirow{2}{*}{\multicolumn{2}{|c|}{ This work }} & USMT-1 & 23.4 & 18.8 & 26.7 & 25.3 & 21.3 & 22.0 \\
\hline & & UNMT-2 & 30.4 & 24.3 & 29.2 & 29.0 & 25.9 & 29.2 \\
\hline \multirow{2}{*}{ (a) } & frequent & USMT-1 & 23.1 & 17.8 & 24.9 & 24.0 & 21.4 & 22.5 \\
\hline & $n$-grams & UNMT-2 & 30.0 & 23.4 & 27.4 & 28.0 & 25.9 & 29.3 \\
\hline \multirow{4}{*}{ (b) } & backward only & USMT-1 & 22.0 & 18.5 & 25.8 & 25.3 & 19.6 & 21.7 \\
\hline & & UNMT-2 & 29.5 & 23.5 & 28.3 & 28.8 & 24.5 & 29.0 \\
\hline & forward only & USMT-1 & 22.5 & 18.6 & 26.3 & 25.7 & 20.4 & 21.5 \\
\hline & lorward ony & UNMT-2 & 29.7 & 24.0 & 28.7 & 29.1 & 25.0 & 29.1 \\
\hline \multirow{2}{*}{ (c) } & not pr & USMT-1 & 23.1 & 18.9 & 26.4 & 25.3 & 21.0 & 22.1 \\
\hline & not prumed & UNMT-2 & 30.4 & 24.5 & 29.3 & 29.0 & 25.8 & 29.0 \\
\hline
\end{tabular}

(a) Phrases: collected with the word2phrase algorithm against using the most frequent $n$ grams, with $n$ up to 3 .

(b) Synthetic parallel data for refinement steps: mixture of synthetic parallel data generated by forward and backward USMT systems against only using synthetic data generated by backward or forward USMT.

(c) Re-trained phrase table: pruned or not using significance testing.

We performed this study on the tasks en-de, en-fr, and en-ja-NTCIR to highlight the impact of our choices where USMT performs reasonably well and for distant languages. The results for USMT, i.e., USMT-1, and UNMT after two training iterations, i.e., UNMT-2, are reported in Table 4.

For (a), we observed that using frequent $n$-grams as phrases to induce the phrase table, instead of phrases extracted via word2phrase, performs lower for en-de and en-fr pairs. The BLEU score of USMT-1 decreases up to 1.8 BLEU points for $\mathrm{fr} \rightarrow \mathrm{en}$. On the other hand, for en-ja-NTCIR, the use of frequent $n$-grams was slightly better, with a gain of 0.5 BLEU points for USMT- 1 en $\rightarrow$ ja. To sum up, the use of frequent $n$-grams was significantly harmful for three translation directions, en $\rightarrow$ de, fr $\rightarrow$ en, and en $\rightarrow$ fr, while we observed only slight improvements or similar BLEU scores for the other three translation directions, de $\rightarrow$ en, ja $\rightarrow$ en, and en $\rightarrow$ ja.

For (b), our results confirmed our assumptions given in Section 3.3: using only synthetic parallel data generated through back-translation, as in previous work, i.e., for which the source side is synthetic, is sub-optimal as many generated source phrases will never be used when decoding manually produced texts. They also skew the probability distribution by considering phrases unseen in the source language. For these reasons, we observed better results using forward translations in five configurations out of six, i.e., except for en $\rightarrow$ ja, thanks to the ability of the language model in dealing with noisy target phrases and in producing locally probable sequences of words.

Finally, for (c), we observed similar BLEU scores with and without pruning the phrase tables trained on synthetic parallel data. In all our configurations, pruning with significance testing removed more than $85 \%$ of the phrase pairs, while the BLEU scores remained stable, highlighting the high ratio of unlikely and noisy phrase pairs learned from the synthetic parallel data.

Interestingly, for all the different configurations, the impact on the translation quality for USMT1 is approximately carried over to UNMT-2. From this empirical observation, we can speculate that the final performance of UNMT-2 is dependent on the performance of the first USMT-1 system and that efforts to improve USMT-1 may lead to similar improvement for UNMT-2. 
Table 5. BLEU Scores With and Without Using Development Data

\begin{tabular}{llcc}
\hline Configuration & \multicolumn{1}{c}{ System } & en $\rightarrow$ fr & en $\rightarrow$ ja-NTCIR \\
\hline \multirow{2}{*}{ USMT-1 } & w/ dev. data & 25.3 & 22.0 \\
& w/o dev. data & 23.4 & 17.2 \\
\hline \multirow{2}{*}{ UNMT-2 } & w/ dev. data & 29.0 & 29.2 \\
& w/o dev. data & 27.2 & 24.5 \\
\hline
\end{tabular}

\subsection{Impact of Using Development Data}

In this work, we assumed the existence of development data for the reasons detailed in Section 3.2 and performed a supervised tuning of USMT and model selection for UNMT.

In this section, to evaluate the impact of this choice on the final result of our approach, we compare two configurations using or not using development data. The configuration using development data is the same one used in previous sections. For the configuration that does not use development data, we perform unsupervised tuning of USMT by using the default weights of Moses and use the UNMT model obtained after 10 training epochs. ${ }^{24}$ We experiment on en $\rightarrow$ fr and en $\rightarrow$ ja-NTCIR and expect to have a larger drop of translation quality for en $\rightarrow$ ja-NTCIR than for $\mathrm{en} \rightarrow \mathrm{fr}$, since previous work has shown that default Moses weights already perform well for en-fr [24].

Results are reported in Table 5 . We observed that while the translation quality decreases by less than 2.0 BLEU points for en $\rightarrow$ fr, it decreases by more than 4.0 BLEU points for en $\rightarrow$ ja-NTCIR. Again, the drop in USMT-1 is carried over UNMT-2. The difference of translation quality between using or not using development data remains the same for USMT-1 and UNMT-2. We can then argue that most of the drop of the translation quality happens due to the unsupervised tuning of USMT with default weights of Moses that are particularly unsuitable for en $\rightarrow$ ja-NTCIR. While the unsupervised tuning method proposed by Artetxe et al. [2] is more elaborated than just using the Moses's default weights, it still depends on them for initialization and we may expect it to perform poorly for en $\rightarrow$ ja-NTCIR. Unsupervised tuning for distant languages remains an open challenge.

\subsection{Impact of the Comparability of Monolingual Corpora}

For en-ja-ALT and ja-id tasks, USMT failed in generating useful synthetic parallel data to initialize our UNMT. The reasons for this failure may be related to the difficulty of the language pairs, since, for instance, English and Japanese are distant languages, relatively for instance to English and German. However, for en-ja-NTCIR we observed that USMT can also generate useful synthetic parallel data for this language pair. Consequently, we hypothesize that, in addition to the language pair, the most important factors to consider for a success in USMT and eventually UNMT are the following two:

(a) the comparability of the L1 and L2 monolingual corpora used for training, and

(b) the similarity between the domain of the monolingual data and the domain of dev/test data.

To test these hypotheses, we performed further experiments in USMT, taking en-fr as an example, by only modifying the combination of monolingual corpora used for phrase table induction and

\footnotetext{
${ }^{24}$ The number of epochs was arbitrarily chosen but we confirmed that it is more epochs than the number of epochs performed with supervised validation.
} 
Table 6. BLEU Scores of USMT for en-fr Obtained Using Different English Monolingual Corpora

\begin{tabular}{lccc}
\hline English corpora & \multicolumn{2}{c}{ BLEU } & English \\
& $\mathrm{fr} \rightarrow \mathrm{en}$ & $\mathrm{en} \rightarrow \mathrm{fr}$ & OOV ratio \\
\hline News Crawl & 26.7 & 25.3 & $7.8 \%$ \\
Common Crawl & 22.5 & 22.6 & $9.5 \%$ \\
NTCIR & 2.5 & 3.2 & $36.9 \%$ \\
\hline
\end{tabular}

The "OOV ratio" is the ratio of the English token types in the test data that are not in the vocabulary of the English monolingual corpus.

during the refinement steps. As English data, we used either News Crawl, Common Crawl, or NTCIR corpora, while the French data remained the News Crawl corpus.

The resulting BLEU scores are summarized in Table 6. We observed that while en-fr can be considered as an easy language pair for USMT, replacing the News Crawl corpus with either a very noisy corpus (Common Crawl) or a clean but out-of-domain corpus (NTCIR) brought a significant drop in translation quality. In particular, the phrase table induced from NTCIR corpus had a very low coverage of the dev/test sets. For instance, in the English side of the test set, $7.8 \%$ (800/10,308), 9.5\% (979/10,308), and 36.9\% $(3,805 / 10,308)$ of the token types were out-of-vocabulary for the phrase tables induced either from English News Crawl, English Common Crawl, or English NTCIR corpora, respectively. In this case, the noisier and smaller Common Crawl corpus was much more helpful than the cleaner and larger but out-of-domain NTCIR corpus.

We also confirmed in additional experiments for en-ja-ALT that none of all the possible combinations of our monolingual corpora achieved better results than using Common Crawl data, while using any other combination of our monolingual corpora than using NTCIR corpora for en-ja-NTCIR led to scores below 5.0 BLEU points for this task.

These results confirm how critical the selection of the monolingual data used to train USMT is, regarding (a) the comparability of the corpora for both languages and (b) the similarity of their domains to the domain of the dev/test data.

\section{FURTHER EXPERIMENTS WITH WIKIPEDIA AS MONOLINGUAL DATA}

From our observations in Section 6.6, we concluded that domain similarity of the monolingual data plays a crucial role in the success of unsupervised MT. In this section, we describe further experiments by using Wikipedia as monolingual data. Wikipedia corpora are especially interesting as we can consider that from one language to another the style of the corpus will remain similar. Moreover, while the Wikipedia corpus is a mixture of many domains, we can expect many articles to have a semantically similar content across languages; some may even be translations. ${ }^{25}$ Given these properties of the Wikipedia corpora, we can expect to achieve a better translation quality than with configurations using very large but less-related monolingual data.

\subsection{Data}

The architectures and hyper-parameters of our MT systems are identical to those used in Section 6. For highlighting the impact of using more comparable monolingual data, we perform new experiments on the three ALT tasks, using Wikipedia corpora instead of the Common Crawl corpora. All the steps of our pipeline, along with the training of the models, such as word embeddings, have been done using Wikipedia corpora.

\footnotetext{
${ }^{25}$ For instance, Schwenk et al. [33] extracted $135 \mathrm{M}$ sentence pairs from Wikipedia that can be used to train NMT systems.
} 
Table 7. Statistics of the Pre-Processed Wikipedia Corpora

\begin{tabular}{lccc}
\hline & English & Indonesian & Japanese \\
\hline \#tokens & $2.8 \mathrm{~B}$ & $71.9 \mathrm{M}$ & $457 \mathrm{M}$ \\
\hline
\end{tabular}

Table 8. Results for the ALT Tasks

\begin{tabular}{lccccccc}
\hline System & & \multicolumn{7}{c}{ ALT } & & \\
& id $\rightarrow$ en & en $\rightarrow$ id & ja $\rightarrow$ en & en $\rightarrow$ ja & id $\rightarrow$ ja & ja $\rightarrow$ id & $\#$ \\
\hline USMT-1 (cc) & 29.1 & 24.5 & 6.0 & 6.4 & 6.6 & 4.4 & 3 \\
UNMT-2 (cc) & 33.0 & 29.3 & 5.4 & 6.9 & 6.9 & 4.8 & 6 \\
\hline USMT-1 (wiki) & 30.8 & 28.1 & 7.5 & 8.6 & 7.7 & 4.7 & 3 \\
$\sqcup$ UNMT-1 (wiki) & 33.3 & 31.0 & 9.3 & 8.6 & 6.6 & 6.6 & 4 \\
$\hookrightarrow$ USMT-2 (wiki) & 31.0 & 30.4 & 8.7 & 6.6 & 6.5 & 4.4 & 5 \\
$\quad \hookrightarrow$ UNMT-2 (wiki) & $\mathbf{3 4 . 0}$ & $\mathbf{3 1 . 3}$ & $\mathbf{9 . 3}$ & $\mathbf{9 . 1}$ & 7.3 & $\mathbf{5 . 0}$ & 6 \\
\hline Supervised SMT & 35.5 & 35.3 & 10.9 & 15.0 & 13.8 & 8.9 & 8 \\
Supervised NMT & 21.6 & 23.4 & 7.1 & 12.1 & 9.8 & 5.0 & 9 \\
\hline
\end{tabular}

Definition of each system (\#ID) follows those in Table 2. "(wiki)" denotes the use of Wikipedia corpora to train the systems, while "(cc)" denotes the use of Common Crawl corpora.

We extracted raw texts from the English, Indonesian, and Japanese Wikipedia dumps of 2017/06/01. Then, we applied the same pre-processing steps described in Section 6.1. Statistics of our Wikipedia data after pre-processing are presented in Table 7. One obvious disadvantage of using these corpora is that they are significantly smaller than the Common Crawl corpora. Smaller training data may lead to much less accurate word embeddings, for instance. We assume that the better comparability of the Wikipedia corpora may compensate this disadvantage to some extent.

\subsection{Results}

As reported in Table 8, the use of Wikipedia corpora brought a better translation quality in all our ALT tasks, despite the use of much smaller monolingual data. Comparing \#6 (cc) and \#6 (wiki), the largest improvement is for ja $\rightarrow$ en, with a gain of 3.9 BLEU points, while ja $\rightarrow$ id remains extremely difficult with only a slight improvement of 0.2 BLEU points. These results confirm that the quality and the comparability of the monolingual corpora used for training unsupervised MT are indeed crucial. We also noticed that improvements observed at USMT-1 (\#3) are carried over UNMT-2 (\#6).

\section{CONCURRENT WORK}

After our preliminary experiments [26], several concurrent works that combine USMT and UNMT or improve UNMT have been published. In this section, we discuss the main similarities and differences between them and ours.

A framework proposed by Ren et al. [31] uses USMT to guide the training of UNMT models in an iterative back-translation process. One of the main differences with our work is that it uses a simpler version of USMT, word-based and without refinement steps, and relies on a unified EM framework for training iteratively USMT and UNMT.

The work in Artetxe et al. [3] is very close to ours. It first proposes to improve USMT using mixed forward and backward translations as synthetic training parallel data for the refinement steps, as we did in Section 3.3, and also proposes, among other modifications, a new feature for the induced phrase table based on the orthographic similarity between the source and target phrases. The resulting USMT system outperforms the USMT system proposed in their previous work [2], 
on European language pairs. However, this work does not provide any ablation study to highlight how the modifications perform individually and we can expect that orthographic similarity features will not be useful, or even misleading, for distant languages that do not share the same writing systems. It also proposes to combine USMT and NMT similarly to what we proposed in [26] by incrementally training NMT on more and more synthetic parallel data. The originality of their approach is that during the training, the training data are progressively switched from data generated by USMT to data back-translated by NMT. They compared with our preliminary results in [26] and reported on higher BLEU scores for the same en-de and en-fr tasks presented in Section 6. However, it remains unclear to what extent their approach depends on the initial quality of the synthetic parallel data generated by USMT. Their proposed UNMT system reaches a significantly better translation quality than ours while they used an NMT system with Transformer "Big" model. For a fair comparison, we consistently used a standard Transformer model (Transformer "Base") and with only four layers to speed up experiments. Last but not least, they did not present any results on truly low-resource and/or distant language pairs or Asian languages.

More recently, a new architecture for pre-training cross-lingual language models, called XLM, has been proposed by Lample and Conneau [22]. In their evaluation, they show that XLM can be used to initialize UNMT training, i.e., using an architecture similar to their previous one but replacing bilingual sub-word embeddings with XLM. They empirically demonstrate that XLM initializes UNMT better than bilingual sub-word embeddings, allowing UNMT to reach a new state-of-theart performance in several translation tasks. In future work, we plan to investigate the promising combination of USMT and UNMT initialized by XLM.

These three works have in common that they performed experiments only on high-resource European language pairs, and with source and target monolingual data that are comparable to some extent. None of them explored the feasibility of their approach in much more difficult settings, i.e., for very distant language pairs and noisy or less-related monolingual corpora, in contrast to this article.

\section{CONCLUSION AND FUTURE WORK}

We proposed a new approach for UNMT that can be straightforwardly exploited with wellestablished architectures and frameworks used for supervised NMT without any modifications. It only assumes for initialization the availability of synthetic parallel data that can be, for instance, generated by USMT. Our approach for unsupervised MT achieved a much higher level of translation quality than previous work. We also showed that ensuring some comparability between the monolingual corpora used for both languages as well as some similarity between their domains and the domain of the dev/test sets is critical for the success of unsupervised MT.

For future work, we will extend our study on the impact of the comparability of monolingual corpora used in unsupervised MT and study methods for selecting useful monolingual corpora without supervision, as well as methods exploiting a weighted combination of out-of-domain and in-domain monolingual corpora. We also plan to propose new applications of our approach in weakly supervised scenarios where a small amount of manually produced parallel sentences is available for training.

\section{ACKNOWLEDGMENTS}

A part of this work was conducted under the program "Research and Development of Enhanced Multilingual and Multipurpose Speech Translation System" of the Ministry of Internal Affairs and Communications (MIC), Japan. A. Fujita is partly supported by JSPS KAKENHI Grant No. 19H05660. We would like to thank all the reviewers for their insightful comments and suggestions. 


\section{REFERENCES}

[1] Mikel Artetxe, Gorka Labaka, and Eneko Agirre. 2018. A robust self-learning method for fully unsupervised cross-lingual mappings of word embeddings. In Proceedings of the 56th Annual Meeting of the Association for Computational Linguistics (Volume 1: Long Papers). Association for Computational Linguistics, 789-798. https:// aclweb.org/anthology/P18-1073.

[2] Mikel Artetxe, Gorka Labaka, and Eneko Agirre. 2018. Unsupervised statistical machine translation. In Proceedings of the 2018 Conference on Empirical Methods in Natural Language Processing. Association for Computational Linguistics, 3632-3642. https://aclweb.org/anthology/D18-1399.

[3] Mikel Artetxe, Gorka Labaka, and Eneko Agirre. 2019. An effective approach to unsupervised machine translation. In Proceedings of the 57th Annual Meeting of the Association for Computational Linguistics. Association for Computational Linguistics, 194-203. https://aclweb.org/anthology/P19-1019.

[4] Mikel Artetxe, Gorka Labaka, Eneko Agirre, and Kyunghyun Cho. 2018. Unsupervised neural machine translation. In Proceedings of the 6th International Conference on Learning Representations. 12. https://openreview.net/forum?id= Sy2ogebAW.

[5] Loïc Barrault, Ondřej Bojar, Marta R. Costa-jussà, Christian Federmann, Mark Fishel, Yvette Graham, Barry Haddow, Matthias Huck, Philipp Koehn, Shervin Malmasi, Christof Monz, Mathias Müller, Santanu Pal, Matt Post, and Marcos Zampieri. 2019. Findings of the 2019 Conference on Machine Translation (WMT19). In Proceedings of the 4th Conference on Machine Translation (Volume 2: Shared Task Papers, Day 1). Association for Computational Linguistics, 1-61. https://aclweb.org/anthology/W19-5301.

[6] Piotr Bojanowski, Edouard Grave, Armand Joulin, and Tomas Mikolov. 2017. Enriching word vectors with subword information. Transactions of the Association for Computational Linguistics 5 (2017), 135-146. https://aclweb. org/anthology/Q17-1010.

[7] Ondřej Bojar, Christian Federmann, Mark Fishel, Yvette Graham, Barry Haddow, Philipp Koehn, and Christof Monz. 2018. Findings of the 2018 conference on machine translation (WMT18). In Proceedings of the 3rd Conference on Machine Translation: Shared Task Papers. Association for Computational Linguistics, 272-303. https://aclweb.org/ anthology/W18-6401.

[8] Yong Cheng, Qian Yang, Yang Liu, Maosong Sun, and Wei Xu. 2017. Joint training for pivot-based neural machine translation. In Proceedings of the 26th International foint Conferences on Artificial Intelligence. International Joint Conferences on Artificial Intelligence, 3974-3980. https://www.ijcai.org/proceedings/2017/0555.pdf.

[9] Colin Cherry and George Foster. 2012. Batch tuning strategies for statistical machine translation. In Proceedings of the 2012 Conference of the North American Chapter of the Association for Computational Linguistics: Human Language Technologies. Association for Computational Linguistics, 427-436. https://aclweb.org/anthology/N12-1047.

[10] Chenchen Ding, Masao Utiyama, and Eiichiro Sumita. 2015. Improving fast_align by reordering. In Proceedings of the 2015 Conference on Empirical Methods in Natural Language Processing. Association for Computational Linguistics, 1034-1039. https://aclweb.org/anthology/D15-1119.

[11] Chris Dyer, Victor Chahuneau, and Noah A. Smith. 2013. A simple, fast, and effective reparameterization of IBM Model 2. In Proceedings of the 2013 Conference of the North American Chapter of the Association for Computational Linguistics: Human Language Technologies. Association for Computational Linguistics, 644-648. https://aclweb.org/ anthology/N13-1073.

[12] Sergey Edunov, Myle Ott, Michael Auli, and David Grangier. 2018. Understanding back-translation at scale. In Proceedings of the 2018 Conference on Empirical Methods in Natural Language Processing. Association for Computational Linguistics, 489-500. https://aclweb.org/anthology/D18-1045.

[13] Isao Goto, Ka Po Chow, Bin Lu, Eiichiro Sumita, and Benjamin K. Tsou. 2013. Overview of the Patent Machine Translation Task at the NTCIR Workshop. In Proceedings of the 10th NTCIR Conference on Evaluation of Information Access Technologies. 260-286. http://research.nii.ac.jp/ntcir/workshop/OnlineProceedings9/NTCIR/01-NTCIR9PATENTMT-GotoI.pdf.

[14] Kenneth Heafield, Ivan Pouzyrevsky, Jonathan H. Clark, and Philipp Koehn. 2013. Scalable modified Kneser-Ney language model estimation. In Proceedings of the 51st Annual Meeting of the Association for Computational Linguistics (Volume 2: Short Papers). Association for Computational Linguistics, 690-696. https://aclweb.org/anthology/P132121.

[15] Liang Huang and David Chiang. 2007. Forest rescoring: Faster decoding with integrated language models. In Proceedings of the 45th Annual Meeting of the Association of Computational Linguistics. Association for Computational Linguistics, 144-151. https://aclweb.org/anthology/P07-1019.

[16] Howard Johnson, Joel Martin, George Foster, and Roland Kuhn. 2007. Improving translation quality by discarding most of the phrasetable. In Proceedings of the 2007 Joint Conference on Empirical Methods in Natural Language Processing and Computational Natural Language Learning (EMNLP-CoNLL). Association for Computational Linguistics, 967-975. https://aclweb.org/anthology/D07-1103. 
[17] Marcin Junczys-Dowmunt, Roman Grundkiewicz, Tomasz Dwojak, Hieu Hoang, Kenneth Heafield, Tom Neckermann, Frank Seide, Ulrich Germann, Alham Fikri Aji, Nikolay Bogoychev, André F. T. Martins, and Alexandra Birch. 2018. Marian: Fast neural machine translation in C++. In Proceedings of ACL 2018, System Demonstrations. Association for Computational Linguistics, 116-121. https://aclweb.org/anthology/P18-4020.

[18] Tomoyuki Kajiwara and Mamoru Komachi. 2016. Building a monolingual parallel corpus for text simplification using sentence similarity based on alignment between word embeddings. In Proceedings of COLING 2016, the 26th International Conference on Computational Linguistics: Technical Papers. The COLING 2016 Organizing Committee, 1147-1158. https://aclweb.org/anthology/C16-1109.

[19] Alexandre Klementiev, Ann Irvine, Chris Callison-Burch, and David Yarowsky. 2012. Toward statistical machine translation without parallel corpora. In Proceedings of the 13th Conference of the European Chapter of the Association for Computational Linguistics. Association for Computational Linguistics, 130-140. https://aclweb.org/anthology/E121014.

[20] Philipp Koehn, Hieu Hoang, Alexandra Birch, Chris Callison-Burch, Marcello Federico, Nicola Bertoldi, Brooke Cowan, Wade Shen, Christine Moran, Richard Zens, Chris Dyer, Ondrej Bojar, Alexandra Constantin, and Evan Herbst. 2007. Moses: Open source toolkit for statistical machine translation. In Proceedings of the 45th Annual Meeting of the Association for Computational Linguistics Companion Volume Proceedings of the Demo and Poster Sessions. Association for Computational Linguistics, 177-180. https://aclweb.org/anthology/P07-2045.

[21] Patrik Lambert, Holger Schwenk, Christophe Servan, and Sadaf Abdul-Rauf. 2011. Investigations on translation model adaptation using monolingual data. In Proceedings of the 6th Workshop on Statistical Machine Translation. Association for Computational Linguistics, 284-293. https://aclweb.org/anthology/W11-2132.

[22] Guillaume Lample and Alexis Conneau. 2019. Cross-lingual language model pretraining. CoRR abs/1901.07291 (2019), 10. http://arxiv.org/abs/1901.07291.

[23] Guillaume Lample, Alexis Conneau, Ludovic Denoyer, and Marc'Aurelio Ranzato. 2018. Unsupervised machine translation using monolingual corpora only. In Proceedings of the 6th International Conference on Learning Representations. 14. https://openreview.net/forum?id=rkYTTf-AZ.

[24] Guillaume Lample, Myle Ott, Alexis Conneau, Ludovic Denoyer, and Marc'Aurelio Ranzato. 2018. Phrase-based \& neural unsupervised machine translation. In Proceedings of the 2018 Conference on Empirical Methods in Natural Language Processing. Association for Computational Linguistics, 5039-5049. https://aclweb.org/anthology/D181549.

[25] Benjamin Marie and Atsushi Fujita. 2018. Phrase table induction using monolingual data for low-resource statistical machine translation. ACM Transactions on Asian and Low-Resource Language Information Processing 17, 3, Article 16 (Feb. 2018), 25 pages. http://doi.acm.org/10.1145/3168054

[26] Benjamin Marie and Atsushi Fujita. 2018. Unsupervised neural machine translation initialized by unsupervised statistical machine translation. CoRR abs/1810.12703 (2018), 13. http://arxiv.org/abs/1810.12703.

[27] Tomas Mikolov, Ilya Sutskever, Kai Chen, Greg S. Corrado, and Jeff Dean. 2013. Distributed representations of words and phrases and their compositionality. In Advances in Neural Information Processing Systems 26, C. J. C. Burges, L. Bottou, M. Welling, Z. Ghahramani, and K. Q. Weinberger (Eds.). Curran Associates, Inc., 3111-3119. https://papers. nips.cc/paper/5021-distributed-representations-of-words-and-phrases-and-their-compositionality.

[28] Franz Josef Och and Hermann Ney. 2002. Discriminative training and maximum entropy models for statistical machine translation. In Proceedings of 40th Annual Meeting of the Association for Computational Linguistics. Association for Computational Linguistics, 295-302. https://aclweb.org/anthology/P02-1038.

[29] Kishore Papineni, Salim Roukos, Todd Ward, and Wei-Jing Zhu. 2002. Bleu: A method for automatic evaluation of machine translation. In Proceedings of 40th Annual Meeting of the Association for Computational Linguistics. Association for Computational Linguistics, 311-318. https://aclweb.org/anthology/P02-1040.

[30] Matt Post. 2018. A call for clarity in reporting BLEU scores. In Proceedings of the 3rd Conference on Machine Translation: Research Papers. Association for Computational Linguistics, 186-191. https://aclweb.org/anthology/W18-6319.

[31] Shuo Ren, Zhirui Zhang, Shujie Liu, Ming Zhou, and Shuai Ma. 2019. Unsupervised neural machine translation with SMT as posterior regularization. In Proceedings of the 33rd AAAI Conference on Artificial Intelligence. Association for the Advancement of Artificial Intelligence, 241-248. https://aaai.org/ojs/index.php/AAAI/article/view/3791.

[32] Hammam Riza, Michael Purwoadi, Gunarso, Teduh Uliniansyah, Aw Ai Ti, Sharifah Mahani Aljunied, Luong Chi Mai, Vu Tat Thang, Nguyen Phuong Thai, Vichet Chea, Rapid Sun, Sethserey Sam, Sopheap Seng, Khin Mar Soe, K hin Thandar Nwet, Masao Utiyama, and Chenchen Ding. 2016. Introduction of the Asian Language Treebank. In Proceedings of the 2016 Conference of the Oriental Chapter of International Committee for Coordination and Standardization of Speech Databases and Assessment Technique (O-COCOSDA). 1-6. https://ieeexplore.iee.org/document/ 7918974.

[33] Holger Schwenk, Vishrav Chaudhary, Shuo Sun, Hongyu Gong, and Francisco Guzmán. 2019. WikiMatrix: Mining 135M parallel sentences in 1620 language pairs from Wikipedia. CoRR abs/1907.05791 (2019), 13. https://arxiv.org/ abs/1907.05791. 
[34] Rico Sennrich, Barry Haddow, and Alexandra Birch. 2016. Improving neural machine translation models with monolingual data. In Proceedings of the 54th Annual Meeting of the Association for Computational Linguistics (Volume 1: Long Papers). Association for Computational Linguistics, 86-96. https://aclweb.org/anthology/P16-1009.

[35] Samuel L. Smith, David H. P. Turban, Steven Hamblin, and Nils Y. Hammerla. 2017. Offline bilingual word vectors, orthogonal transformations and the inverted softmax. In Proceedings of the 5th International Conference on Learning Representations. 10. https://openreview.net/forum?id=r1Aab85gg.

[36] Anders Søgaard, Sebastian Ruder, and Ivan Vulić. 2018. On the limitations of unsupervised bilingual dictionary induction. In Proceedings of the 56th Annual Meeting of the Association for Computational Linguistics (Volume 1: Long Papers). Association for Computational Linguistics, 778-788. https://aclweb.org/anthology/P18-1072.

[37] Yangqiu Song and Dan Roth. 2015. Unsupervised sparse vector densification for short text similarity. In Proceedings of the 2015 Conference of the North American Chapter of the Association for Computational Linguistics: Human Language Technologies. Association for Computational Linguistics, 1275-1280. https://aclweb.org/anthology/N15-1138.

[38] Nicola Ueffing, Gholamreza Haffari, and Anoop Sarkar. 2007. Transductive learning for statistical machine translation. In Proceedings of the 45th Annual Meeting of the Association of Computational Linguistics. Association for Computational Linguistics, 25-32. https://aclweb.org/anthology/P07-1004.

[39] Ashish Vaswani, Noam Shazeer, Niki Parmar, Jakob Uszkoreit, Llion Jones, Aidan N. Gomez, Łukasz Kaiser, and Illia Polosukhin. 2017. Attention is all you need. In Advances in Neural Information Processing Systems 30. Curran Associates, Inc., 5998-6008. https://papers.nips.cc/paper/7181-attention-is-all-you-need.

[40] Zhen Yang, Wei Chen, Feng Wang, and Bo Xu. 2018. Unsupervised neural machine translation with weight sharing. In Proceedings of the 56th Annual Meeting of the Association for Computational Linguistics (Volume 1: Long Papers). Association for Computational Linguistics, 46-55. https://aclweb.org/anthology/P18-1005.

[41] Richard Zens, Daisy Stanton, and Peng Xu. 2012. A systematic comparison of phrase table pruning techniques. In Proceedings of the 2012 Joint Conference on Empirical Methods in Natural Language Processing and Computational Natural Language Learning. Association for Computational Linguistics, 972-983. https://aclweb.org/anthology/D121089.

[42] Biao Zhang, Deyi Xiong, and Jinsong Su. 2018. Accelerating neural transformer via an average attention network. In Proceedings of the 56th Annual Meeting of the Association for Computational Linguistics (Volume 1: Long Papers). Association for Computational Linguistics, 1789-1798. https://aclweb.org/anthology/P18-1166.

[43] Kai Zhao, Hany Hassan, and Michael Auli. 2015. Learning translation models from monolingual continuous representations. In Proceedings of the 2015 Conference of the North American Chapter of the Association for Computational Linguistics: Human Language Technologies. Association for Computational Linguistics, 1527-1536. https:// aclweb.org/anthology/N15-1176.

Received July 2019; revised January 2020; accepted March 2020 